\title{
Variabilité du pouvoir pathogène chez Septoria nodorum Berk. (Leptosphaeria nodorum Müll.)
}

\author{
M. Skajennikoff et F. Rapilly \\ INRA, station de pathologie végétale. 78026 Versailles Cedex, France
}

(reçu le 23 septembre 1988, accepté le 24 avril 1989)

\begin{abstract}
Résumé - Les résultats consignés montrent que ies amplitudes de variation du pouvoir pathogène de Septoria nodorum, évaluées par la réduction du poids de 1000 grains ou par des éléments de la résistance partielle, sont du même ordre de grandeur entre isolats, entre pycnides issues d'un même isolat, qu'entre monopycnidiospores issues d'un même cirrhe. Les souches de $S$. nodorum sont donc des populations faites d'éléments aux pouvoirs pathogènes différents.

Le noyau et le cytoplasme de l'hôte exercent sur ces populations des pressions de sélection permettant aux éléments les mieux adaptés à l'hôte de passage d'être sélectionnés.

Suivant le niveau des perturbations qu'ils provoquent sur les blés sensibles, on peut classer les cytoplasmes dans l'ordre suivant: Triticum timopheevi > Secale cereale $>T$. aestivum. Sur le plan de la dynamique de l'épidémie, ces perturbations peuvent se traduire par une moindre compétitivité des souches. La réponse de lignées de blé à haut niveau de résistance générale ne semble pas perturbée. Toutefois, le gain d'agressivité qui s'exprime sur l'hôte de passage, dès le premier passage, tend à s'amenuiser au fur et à mesure des passages successifs.
\end{abstract}

blé - Triticale - pression de sélection, isolat - monopycnidiospore

Summary - Variability of pathogenicity in Septoria nodorum Berk. (= Leptosphaeria nodorum Müll.). The parasite can adjust its pathogenicity by means of two mechanisms: one is at the level of ascospores differenciation, the other is the result of heterogenicity among the spores resulting from vegetative multiplication of the fungus. These two mechanisms participate in the initiation and spreading of epidemics. This study will deal with the second mechanism.

The following material was used: 1) host range (Table I) : two wheat cultivars, two series of triticales and one rye cultivar; and 2) fungal isolates : isolate 6 (wheat adapted), isolate 42 (triticale adapted), monopycnidiospore cultures derived from 6 and 42 and subcultures derived from a single spore, isolate collected in Lyon's area-strain "Lyon" (Rhône-Poulenc), isolates collected in Israel : strains $I S$, and $I S_{2 .}$ - The following measurements were made: 1) thousand-grain-weight (PMG) or reduction of thousand-grain-weight (RPMG), 2) rate of spread of foliar necrosis ( $\left.P_{\max }\right)$, 3) rate of spread of epidemics.

The selection pressure by host-plants was obtained with 1-4 passages through the host, using inoculation of detached seedling leaves. The parasite was re-isolated from pycnidia and inoculated on ears.

Significant differences in aggressiveness, as expressed by thousand-grain-weight, were observed between pycnidia cultures isolated from the same strain (Table II), between monopycnidiospore cultures derived from a same pycnidium (Tables III, IV) or between 2 generation monopycnidiospore subcultures (Table V). Similar observations were made with tests on Triticale "Clercal" (Table VI).

The difference between the dynamics of strains 6 and 42 was revealed by two tests: Fig. 1. : on two wheat lines, the one susceptible, the other with a high partial resistance level; and Fig. 2. : on two susceptible wheat cultivars, according to the number of dissemination and contamination periods.

Variability of aggressiveness of pathogen strains towards their own hosts, was also expressed by thousand-grainweight in response to selection pressure by several alien hosts (Table VIla). The range of isolates is not the same after host passaging (Table VIIb). The particular effect of genotype and cytoplasm was illustrated with series of triticale: one isoplasmic with various genomes, the other isogenic and alloplasmic (Table VIII, Fig. 3).

The effect of number of passages (1-4) of strain 6 through an alloplasmic list of three Triticale, observed either on two susceptible wheat cultivars (Fig. 3) or on passaging host (Fig. 4), showed that the increase in pathogenicity occurred from the first passage onwards.

Concerning the partial resistance we observed that the passage of strain 6 through the Triticale "Clercal" reduced the incubation time $(L P I)$ and increased the rate of spread of foliar necrosis $\left(P_{\text {max }}\right)$.

These parasite variations led to an increased pathogenicity on susceptible varieties, but the behaviour of lines with a high level of general resistance remained constant (Table IX). 
All observations confirm that every Septoria nodorum isolate is a population possessing a great flexibility, on which a host-plant can exert a selection pressure by means of its genotype and (or) its cytoplasm.

As the difference between the genotype or cytoplasm of alien hosts and those of cultivated wheat increases, the resulting re-isolate bec:omes more agressive towards these wheats.

Finally, the expression of the aggressiveness observed on ears or foliage after direct contamination should be distinguished from epidemical progression.

Our results concerning the second generation monospores cultures seem to disagree with those of Osbourn (1985). Is the aggressiveness governed by the nucleus alone, by the cytoplasmic genome or by the nucleo-cytoplasmic interactions?

It seemed therefore necessary to study the pathogenicity of Septoria nodorum ascospores resulting from karyogamy in order to gain a better understanding of its capacity for adaptation.

wheat - Triticale - selection pressure - isolate - monopycnidiospore

\section{INTRODUCTION}

La sélection des variétés de blé pour la résistance à Septoria nodorum est orientée vers deux voies. La première, basée sur l'exploitation de la tolérance, prend comme critère la réduction du poids de 1000 grains après contamination des plantes à l'anthèse; c'est l'orientation retenue par Bronnimann (1968), Scott (1973) et Trottet et al. (1975). La deuxième s'appuie sur la résistance partielle du feuillage, qui permet de maintenir l'épidémie en dessous de son seuil de nuisibilité (Rapilly et al. (1984). Nous avons montré que ces deux orientations font appel chez l'hôte à des mécanismes héréditaires impliquant des chromosomes différents (Rapilly et al., 1988a). Cependant, le sélectionneur doit pouvoir les associer dans ses programmes.

Quelle que soit la démarche retenue, son intérêt dépend, à long terme, des conséquences épidémiologiques induites par les capacités du parasite à contourner ces mécanismes de tolérance et/ou de résistance partielle. Nous avons montré par la simulation (Rapilly \& Delhotal, 1986) que l'on pouvait atteindre des niveaux de résistance suffisants pour des conditions climatiques très variées. La simulation a montré également qu'un parasite ne peut être à l'origine d'une épidémie que s'il possède un niveau d'agressivité élevé au moment de la contamination primaire.

Deux mécanismes permettent la variation du pouvoir pathogène de ce parasite. Le premier est lié à la différentiation des ascospores, impliquant l'intervention de la recombinaison méiotique. Rappelons que Septoria nodorum est un champignon hétérothallique (Rapilly et al., 1973; Halama \& Lacoste, 1987), dont les ascospores parvenues à maturité au début de la végétation de l'hôte participent à l'initiation des épidémies. Le second est lié à l'hétérogénéité que l'on observe entre les spores issues de la multiplication végétative de ce champignon. Les hôtes exercent sur le parasite des pressions de sélection (Krupinsky, 1982; Cunfer \& Youmans, 1983), qui se tradui- sent par une augmentation ou une réduction de son pourvoir pathogène (Skajennikoff \& Rapilly, 1983; Osbourn, et al., 1986).

Cette étude a pour objet d'apprécier la variabilité potentielle de la forme de multiplication asexuée de $S$. nodorum. Afin de juger des effets dissociés ou associés du génotype et du cytoplasme de l'hôte sur le pouvoir pathogène du parasite, nous avons retenu la mesure de la réduction du poids de mille grains (en \%) ou un élément de la résistance partielle. Nous avons fait l'hypothèse que des souches de $S$. nodorum provenant d'aires géographiques et d'hôtes différents n'ont pas le même pouvoir pathogène.

\section{MATÉRIEL ET MÉTHODES}

\section{Matériel végétal}

Celui-ci appartient à 3 genres (récapitulatif Tableau I). Les variétés de blé "Etoile de Choisy" et "Roazon" nous ont servi le plus souvent d'hôtes pour apprécier le pouvoir pathogène des diverses souches du parasite.

Les Triticale ont permis de constituer deux séries:

- une première série isoplasmique sur cytoplasme de T. timopheevi comprend le T. 26, dont le génotype est celui de "Clercal» $=$ T. 532 , et le T. 762, dont le génotype correspond à la lignée T. 533-8, lignée sœur de "Clercal»;

- la deuxième série a le génotype de T. 533-8, sur 3 cytoplasmes différents: $T$. aestivum pour T. 533-8, T. timopheevi pour T. 762 et Secale cereale pour T. 832. L'origine de cette série alloplasmique isogénique est décrite par Cauderon et al. (1985).

Le troisième genre est représenté par la variétépopulation de seigle "Petkus".

\section{Souches du parasite}

Les souches de Septoria nodorum étudiées sont les isolats 6 (isolé de blé cultivar "Etoile de Choisy") et 42 (isolé de Triticale cultivar "Clercal»), déjà décrits dans diverses études, dont Skajennikoff \& Rapilly (1985). A partir de ces souches, des cultures monopycnidiospores ont été obtenues en prélevant des spores iso- 
Tableau I. Récapitulatif des hôtes utilisés.

\begin{tabular}{cllll}
\hline \multicolumn{1}{c}{ Blé } & & Triticale & & \multicolumn{1}{c}{ Seigle } \\
& & & & \\
& Variété & Cytoplasme & Génotype & \\
- cv. «Etoile de Choisy», “Roazon» & $T .26$ & $T$. timopheevi & $T .532$ & cv Petkus variété-population \\
- Lignées en cours de sélection: & $T .762$ & T. timopheevi & $T .533-8$ & \\
1/ L22 (Rapilly et al., 1984) & $T .532$ Clercal & T. aestivum & $T .532$ & \\
2/ Lignées programme INRA-Club & $T .533$ & T. aestivum & $T .533-8$ & \\
des cinq: B1, etc... & $T .832$ & Secale cereale & $T .533-8$ & \\
\hline
\end{tabular}

lées après étalement d'un cirrhe. Dans ce cas et d'après les travaux de Shaw (1953) confirmés par ceux de Newton \& Caten (1985), ces cultures monopycnidiospores sont, sur le plan nucléaire, des clones: les noyaux d'une spore étant tous identiques entre eux. A partir d'une culture monopycnidiospore, des sous-cultures monospores sont prélevées qui, théoriquement, sont toutes identiques entre elles sur le plan nucléaire.

Trois autres souches isolées de blé ont également été utilisées. L'une (Souche «Lyon») fournie par la société Rhône-Poulenc et les deux autres (Souches IS1 et IS2) fournies par le Dr. Dinoor (Israël).

Tous les isolats (populations ou monopycnidiospores) sont conservés sur grains de blé, Triticale ou seigle, après contamination des épis au stade anthèse à l'aide de cultures sporulantes obtenues sur milieu gélosé enrichi de $2 \%$ de malt.

\section{Appréciations du pouvoir pathogène}

Toutes les contaminations ont été faites par pulvérisations de suspensions de spores titrant $10^{6} \mathrm{ml}^{-1}$, soit sur plantes en végétation au champ (stades gonflement et anthèse), soit sur plantules cultivées en chambre climatique (stade 3 feuilles), soit encore par dépôt d'une goutte de suspension de spores sur des feuilles détachées, maintenues en survie sur benzimidazole à $50 \mathrm{ppm}$. Pour respecter les 2 voies prises par les sélectionneurs, les appréciations ont porté sur :

Poids de 1000 grains (P.M.G.) ou la réduction de celui-ci (RPMG) après contamination de l'épi à l'anthèse

Dans quelques cas, le taux de fontes de semis résultant de ces contaminations a été mesuré.

\section{Mesure de l'extension des nécroses foliaires $\left(P_{\max }\right)$}

La mesure a lieu $7 \mathrm{j}$ après la contamination de feuilles détachées (ou de plantules) maintenues à $17^{\circ} \mathrm{C}$. Ce paramètre de résistance partielle foliaire est le plus important pour expliquer la progression des épidémies.

\section{Vitesse d'évolution d'épidémies provoquées au champ, sur des microparcelles}

Deux évolutions d'épidémies ont été comparées:

- sur plusieurs lignées inoculées au stade "gonfle- ment» avec les deux souches 6 et 42 apportées séparément,

- sur une même lignée inoculée à 3 stades (deux nœuds, mi-montaison et début épiaison), les deux souches étant apportées séparément ou en mélange.

L'évolution, au cours du temps, de la surface foliaire malade totale est périodiquement appréciée. Les comparaisons entre les épidémies sont faites à l'aide de la représentation des aires sous la courbe (ADPC = Area Disease Progress Curve) délimitée par l'évolution du $\%$ de surface foliaire malade (LAIFM) en fonction du temps.

\section{Pressions de sélection exercées par les hôtes}

Cette étude est réalisée par inoculation des feuilles maintenues en survie. Après apparition des pycnides sur les nécroses, le parasite est ré-isolé, puis, après multiplication sur un milieu gélosé, la nouvelle souche sert à inoculer l'épi d'un hôte ou à contaminer une autre feuille. Suivant les cas, de 1 à 4 passages successifs sur feuilles sont réalisés avant de contaminer les épis soit de l'hôte de passage, soit d'une variété de blé choisie comme référence.

\section{Interprétation des résultats}

Ces expérimentations ont été conduites au cours de 3 années consécutives, mais la variabilité annuelle induite par le climat ne permet pas de faire des comparaisons entre celles-ci. Pour chaque essai, les répétitions, au nombre de 5 à 7 , suivant les cas, ont permis d'estimer la signification des résultats à l'aide d'analyses statistiques (analyse de variance, p.p.d.s.). Pour les épidémies provoquées au champ, le critère retenu est l'aire délimitée par la représentation graphique de l'évolution du p. 100 de surface foliaire malade et l'axe du temps (ADPC).

\section{RÉSULTATS}

Variabilité observée sans pression de sélection

\section{Par rapport aux poids de 1000 grains (PMG et RPMG)}

\section{Variations entre pycnides d'une même souche}

Nous avons comparé le pouvoir pathogène de souches issues de pycnides différentes provenant d'une même culture 42 , cela sur deux varié- 
tés (Tableau II). Nous constatons au seuil de $10 \%$ que le pouvoir pathogène moyen des spores contenues dans le cirrhe $\mathrm{C} 4$ est inférieur à celui des 2 autres cirrhes. Les faibles différences enregistrées illustrent le fait que chaque pycnide est une population.

\section{Variations entre monopycnidiospores issues d'une même pycnide}

Cette comparaison a été faite pour les souches 6 et 42 . II existe pour ces 2 souches des différences significatives entre cultures monospores issues d'une même pycnide (Tableau III, IV). Sui-

Tableau II. Variation de la réduction du poids de 1000 grains exprimée en \% pour deux variétés de blé, contaminées par des cultures monopycnides provenant de la souche 42 .

\begin{tabular}{|c|c|c|c|c|c|}
\hline \multirow[t]{2}{*}{$\begin{array}{c}\text { Variétés de } \\
\text { Blé }\end{array}$} & \multicolumn{3}{|c|}{$\begin{array}{c}\text { Pycnides prélevées sur } \\
\text { la souche } 42\end{array}$} & \multirow[t]{2}{*}{$\begin{array}{c}\text { Population } \\
42\end{array}$} & \multirow[t]{2}{*}{$\begin{array}{c}\text { Moyenne } \\
\text { des pycnides }\end{array}$} \\
\hline & $\mathrm{C} 4$ & $\mathrm{C} 5$ & C6 & & \\
\hline Etoile de & 17,61 & 22,38 & 21,20 & 16,67 & 20,40 \\
\hline Choisy & a & b & b & & \\
\hline Roazon & 17,90 & 19,54 & 24,85 & 21,31 & 20,76 \\
\hline
\end{tabular}

Les lettres indiquent, pour chaque ligne, si les valeurs sont différentes entre elles au seuil de $5 \%$. vant les hôtes de lecture, on peut regrouper ces cultures monospores en 3 ou 4 groupes ayant des niveaux d'agressivité différents. Ces différences s'observent malgré les variations interannuelles (1P). Enfin, on note parfois des inversions de réponse des hôtes aux monopycnidiospores; c'est le cas des cultures C6-3 et C5-3. II faut aussi relever le peu de concordance entre le RPMG et le taux de fonte de semis. Pour ce dernier paramètre, les monospores diffèrent aussi entre-elles, cas des C5-3 et C5-20 lues sur le cultivar «Roazon».

\section{Variations entre monopycnidiospores issues d'une même monopycnidiospore}

Entre monopycnidiospores de la deuxième génération, il existe encore des différences significatives (Tableau V). Si, sur «Roazon", la variation de ces monopycnidiospores ufilles" est moins grande (de 1 à 1,9 ), elle est, sur «Etoile de Choisy", du même ordre que celle obtenue avec les monopycnidiospores de première génération (de 1 à 3,4$)$.

\section{Critères épidémiques}

\section{Extension des nécroses foliaires}

Dans le tableau VI est reportée la taille des nécroses observées sur le Triticale et «Clercal»,

Tableau III. Réduction en \%, en 1984 et en 1985, du poids de 1000 grains de deux variétés de blé après contamination à l'anthèse par des cultures monopycnidiospores, issues d'une même pycnide de la souche 6 . Pour l'année 1984 est indiqué, dans quelques cas, le \% de fontes de semis affectant les grains malades.

Résultats 1984: Monopycnidiospores issues du cirrhe C5, souche 6

\begin{tabular}{|c|c|c|c|c|c|c|c|c|c|c|c|c|c|}
\hline Variétés & pulation & $C 5-1$ & $C 5-2$ & $C 5-3$ & $C 5-6$ & C5-8 & C5-12 & C5-16 & C5-18 & 3 C5-20 & C5-24 & F. Cal & Moyenne \\
\hline $\begin{array}{l}\text { Etoile } \\
\text { de } \\
\text { Choisy }\end{array}$ & 12,11 & $\begin{array}{l}15,79 \\
(1) \mathrm{cd} \\
(2)-\end{array}$ & $\begin{array}{l}16,84 \\
\text { cd } \\
-\end{array}$ & $\begin{array}{l}28,36 \\
a \\
20\end{array}$ & $\begin{array}{l}13,73 \\
d \\
-\end{array}$ & $\begin{array}{l}21,34 \\
b c \\
-\end{array}$ & $\begin{array}{l}20,54 \\
\text { bc } \\
-\end{array}$ & $\begin{array}{l}22,52 \\
b c \\
22\end{array}$ & $\begin{array}{l}16,64 \\
\text { cd } \\
-\end{array}$ & $\begin{array}{l}25,41 \\
a b \\
12\end{array}$ & $\begin{array}{l}20,03 \\
b c \\
25\end{array}$ & $19,46 \mathrm{HS}$ & 20,12 \\
\hline Roazon & 11,26 & $\begin{array}{l}10,81 \\
(1) \mathrm{cd} \\
(2)-\end{array}$ & $\begin{array}{l}18,28 \\
a b \\
--\end{array}$ & $\begin{array}{l}9,61 \\
d \\
56\end{array}$ & $\begin{array}{l}14,39 \\
b c \\
-\end{array}$ & $\begin{array}{l}19,07 \\
\mathrm{a} \\
55\end{array}$ & $\begin{array}{l}12,74 \\
c \\
-\end{array}$ & $\begin{array}{l}\text { 4,97 } \\
\text { c } \\
44\end{array}$ & $\begin{array}{l}11,72 \\
\text { bcde } \\
-\end{array}$ & $\begin{array}{l}13,01 \\
\mathrm{~cd} \\
30\end{array}$ & $\begin{array}{l}8,30 \\
\mathrm{e}^{8} \\
39\end{array}$ & $15,76 \mathrm{HS}$ & 12,29 \\
\hline
\end{tabular}

Résultats 1985: Monopycnidiospores issues du cirrhe 1P, souche 6.

\begin{tabular}{|c|c|c|c|c|c|c|c|c|c|c|c|c|c|c|}
\hline Variétés & Population & $61 P 1$ & $1 P 2$ & $1 P 3$ & $1 P 4$ & $1 P 5$ & $1 P 6$ & $1 P 7$ & $1 P 8$ & $f P 9$ & $1 P 10$ & $1 P 11$ & $1 P 12$ & F. Cal Moyenne \\
\hline \multirow{2}{*}{$\begin{array}{l}\text { Etoile } \\
\text { de } \\
\text { Choisy }\end{array}$} & \multirow[t]{2}{*}{16,6} & 15,67 & 10,14 & 7,16 & 3,04 & 12,44 & 8,19 & 14,32 & 13,90 & 12,04 & 8,28 & 10,95 & 11,47 & \multirow[t]{2}{*}{18,4 HS 10,63} \\
\hline & & (1) $\mathrm{a}$ & $\mathrm{cd}$ & d & e & $\mathrm{c}$ & d & $a b$ & $a b c$ & c & $d$ & $\mathrm{~cd}$ & c & \\
\hline \multirow{2}{*}{ Roazon } & \multirow{2}{*}{11,26} & 9,38 & 16,97 & 15,24 & 14 & 17,38 & 14,17 & 17,73 & 17,44 & 17,12 & 18,12 & 12,87 & 11,18 & \multirow{2}{*}{$5,32 \mathrm{~S} \quad 15,13$} \\
\hline & & (1) e & $a b$ & $\mathrm{bc}$ & $\mathrm{bc}$ & $a b$ & $\mathrm{bc}$ & $a b$ & $a b$ & $a b$ & a & $\mathrm{cd}$ & de & \\
\hline
\end{tabular}

(1) Les lettres indiquent au seuil de $5 \%$ et pour une variété les différences siginficatives entre réduction du poids de 1000 grains.

(2) Taux en \% de fonte de semis. 
Tableau IV. Réduction en \% (année 1984) du poids de 1000 grains de deux variétés de blé, après contamination à l'anthèse par des cultures monopycnidiospores issues d'une même pycnide de la souche 42 . Dans quelques cas est indiqué le \% de fontes de semis affectant les grains malades.

Résultats 1984: Monopycnidiospores issues du cirrhe C6, souche 42.

\begin{tabular}{|c|c|c|c|c|c|c|c|c|c|c|c|}
\hline Variétés & Population 42 & C6-1 & $C 6-3$ & $C 6-9$ & C6-10 & C6-11 & C6-19 & C6-20 & C6-21 & F. Cal & Moyenne \\
\hline $\begin{array}{l}\text { Etoile } \\
\text { de } \\
\text { Choisy }\end{array}$ & 17,89 & $\begin{array}{l}18,45 \\
(1) \mathrm{C} \\
(2)-\end{array}$ & $\begin{array}{l}24,83 \\
b \\
33\end{array}$ & $\begin{array}{l}22,58 \\
b c \\
-\end{array}$ & $\begin{array}{l}19,90 \\
b \\
23\end{array}$ & $\begin{array}{l}23,42 \\
b c \\
25\end{array}$ & $\begin{array}{l}19,78 \\
c \\
-\end{array}$ & $\begin{array}{l}29,25 \\
\mathrm{a} \\
23\end{array}$ & $\begin{array}{l}11,88 \\
d \\
22\end{array}$ & $20,95 \mathrm{HS}$ & 21,26 \\
\hline Roazon & 14,93 & $\begin{array}{l}23,12 \\
(1) b \\
(2)-\end{array}$ & $\begin{array}{l}14,61 \\
c \\
79\end{array}$ & $\begin{array}{l}20,86 \\
b c \\
-\end{array}$ & $\begin{array}{l}24,34 \\
b \\
80\end{array}$ & $\begin{array}{l}34,5 \\
a \\
85\end{array}$ & $\begin{array}{l}32,44 \\
\text { a } \\
-\end{array}$ & $\begin{array}{l}31,24 \\
a \\
80\end{array}$ & $\begin{array}{l}17,71 \\
c \\
78\end{array}$ & $52,90 \mathrm{HS}$ & 24,85 \\
\hline
\end{tabular}

(1) Les lettres indiquent au seuil de $5 \%$ et pour une variété les différences significatives entre réductions du poids de 1000 grains.

(2) Taux en $\%$ de fonte de semis.

Tableau V. Réduction en \%, du poids de 1000 grains des variétés «Etoile de Choisy» et «Roazon» après contamination par des monopycnidiospores «filles» issues de la monopycnidiospore 1P3 de la souche 6 (année 1986).

Résultats 1986: monopycnidiospores «filles» issues de la monopycnidiospore 1P3 de 1985.

\begin{tabular}{|c|c|c|c|c|c|c|c|c|c|c|c|c|c|c|c|}
\hline Variétés & Jation & $1 P 3-1$ & $1 P 3-2$ & 1P3-3 & $1 P 3-4$ & $1 P 3-5$ & $1 P 3-6$ & $1 P 3-7$ & $1 P 3-8$ & 1P3-9 & $1 P 3-10$ & $1 P 3-11$ & 1P3-12 & F. Cal. & Moyenne \\
\hline $\begin{array}{l}\text { Etoile de } \\
\text { Choisy }\end{array}$ & 17,20 & $\begin{array}{l}7,16 \\
\text { (1)ab }\end{array}$ & $\begin{array}{l}6,42 \\
a b\end{array}$ & $\begin{array}{l}7,03 \\
a b\end{array}$ & $\begin{array}{l}2,77 \\
a\end{array}$ & $\begin{array}{l}4,82 \\
a b\end{array}$ & $\begin{array}{l}5,78 \\
a b\end{array}$ & $\begin{array}{l}6,78 \\
a b\end{array}$ & $\begin{array}{l}6,27 \\
a b\end{array}$ & $\begin{array}{l}3,90 \\
a b\end{array}$ & $\begin{array}{l}5,62 \\
a b\end{array}$ & $\begin{array}{l}4,97 \\
a b\end{array}$ & $\begin{array}{l}9,48 \\
b\end{array}$ & 3,65 & 5,91 \\
\hline paz & 14,26 & $\begin{array}{l}7,59 \\
\text { (1)a }\end{array}$ & $\begin{array}{l}13,43 \\
b c\end{array}$ & $\begin{array}{l}7,14 \\
a\end{array}$ & $\begin{array}{l}10,32 \\
a b\end{array}$ & $\begin{array}{l}11,66 \\
b c\end{array}$ & $\begin{array}{l}11,82 \\
\text { bc }\end{array}$ & $\begin{array}{l}12,63 \\
b c\end{array}$ & $\begin{array}{l}12,03 \\
b c\end{array}$ & $\begin{array}{l}11,76 \\
\text { bc }\end{array}$ & $\begin{array}{l}9,26 \\
a b\end{array}$ & $\begin{array}{l}13,37 \\
b c\end{array}$ & $\begin{array}{l}12,38 \\
\text { bc }\end{array}$ & 3,385 & 11,11 \\
\hline
\end{tabular}

(1) Les lettres indiquent au seuil de $5 \%$ et pour une variété les diftérences significatives entre réductions de poids de 1000 grains.

après contamination par des cultures issues de monopycnidiospores prélevées sur diverses pycnides de la souche 6 . Là aussi, on constate des différences qui confirment que, pour ce critère, le contenu d'une pycnide est hétérogène. Nous avons aussi noté que les délais nécessaires à la production des pycnides sur ces nécroses varient de 10 à 15 j suivant la monopycnidiospore.

\section{Progression des épidémies: A.D.P.C.}

L'inoculation de 20 lignées de blé par la souche 6 , d'une part, et par la souche 42 , d'autre part, nous a permis de comparer les capacités épidémiques de ces 2 souches. Pour les 20 lignées confondues, la comparaison des aires totales sous les courbes montre que, si les lignées diffèrent entre elles (F. cal. = 9,01 H.S.), les souches, elles, donnent globalement une même réponse.

Cependant, une étude plus attentive illustrée par la Figure 1, sur laquelle sont représentées les évolutions des 2 souches sur 1 lignée sensible (lignée I) et sur une lignée ayant un haut niveau de résistance partielle (lignée II), permet de diviser les aires en3 zones:

- la zone A, correspondant à la maladie issue directement de l'inoculation,
- la zone B, qui correspond, d'une part, à l'inoculation directe et, d'autre part, aux premières recontaminations qui lui succèdent,

- la zone $C$, qui n'a pour origine que les contaminations secondaires du parasite.

Tableau VI. Variation de la taille de la nécrose foliaire sur le Triticale "Clercal», après contamination par 4 cultures monopycnidiospores provenant de plusieurs pycnides de la souche 6 . Taille en $\mathrm{mm}$ après $7 \mathrm{j}$ à $17^{\circ} \mathrm{C}$ (valeur moyenne de 15 à 20 répétitions).

\begin{tabular}{clllll}
\hline \multirow{2}{*}{$\begin{array}{c}\text { mopycni- } \\
\text { diospores }\end{array}$} & \multicolumn{5}{c}{ Pycnides (souche 6) } \\
& & $P 2$ & $P 3$ & $P 4$ & $P 5$ \\
\hline \multirow{2}{*}{1} & 8,06 & 6,87 & 8,44 & 4,1 & 3,7 \\
& $\mathrm{a}$ & $\mathrm{a}$ & $\mathrm{a}$ & $\mathrm{a}$ & $\mathrm{a}$ \\
2 & 7,39 & 5,64 & 7,47 & 6,07 & 6,66 \\
& $\mathrm{a}$ & $\mathrm{ab}$ & $\mathrm{a}$ & $\mathrm{a}$ & $\mathrm{b}$ \\
3 & 8 & 5 & 7,41 & 4,84 & 8,53 \\
& $\mathrm{a}$ & $\mathrm{ab}$. & $\mathrm{a}$ & $\mathrm{a}$ & $\mathrm{b}$ \\
4 & 6,68 & 4,27 & 3,69 & - & 7,33 \\
& $\mathrm{a}$ & $\mathrm{b}$ & $\mathrm{b}$ & & $\mathrm{b}$ \\
moyenne & 7,53 & 5,44 & 6,75 & 5 & 6,55 \\
\hline
\end{tabular}

Les lettres indiquent si les valeurs d'une même colonne sont diffrentes entre elles au seuil de $5 \%$. 


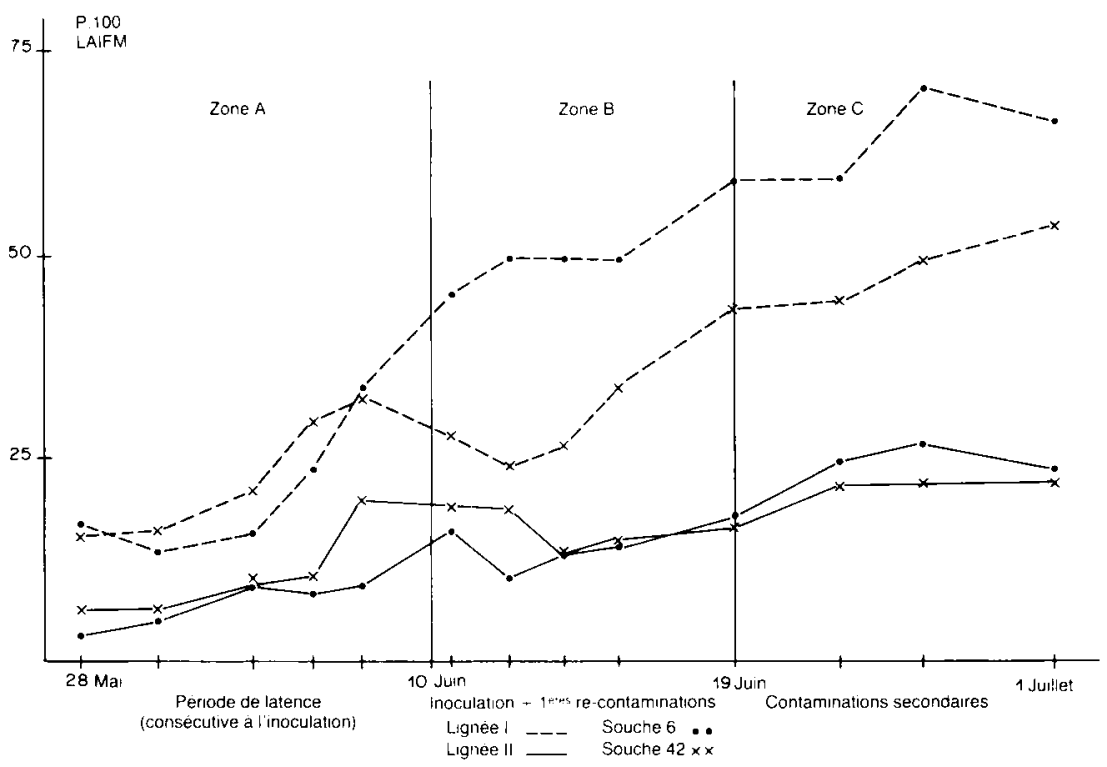

Fig. 1. Evolution d'épidémies de Septoriose initiées avec la souche 6 (..) ou la souche 42 ( $x x)$ sur une lignée très sensible $A(--$.$) ou$ sur une lignée à résistance partielle élevée B (-

Les analyses faites sur ces 3 zones montrent que:

- il y a inversion du comportement des isolats entre début et fin d'épidémie, l'isolat 6 produisant moins de surface malade en début d'épidémie et davantage en fin d'épidémie que l'isolat 42 . Pour la zone A: $F$. Cal. $=5,225$, pour la zone B: $F$. Cal. $=4,855$;

- cette inversion se fait plus tardivement dans le cas d'une lignée à résistance partielle (II) que pour une lignée sensible (I);

- les différences observées entre isolats en fin d'épidémie et non révélées par les autres appréciations sont d'autant plus marquées que la lignée hôte est sensible.

Deux observations similaires faites, une autre année, sur 2 cultivars sont illustrées par les Figures 2 ( $A-B$ et $C$ ). Ici, la population hôte est la même et c'est le stade d'initiation de l'épidémie qui diffère. On constate que plus la durée de l'épidémie est prolongée et que, par conséquent, la fréquence des périodes successives de dissémination et de contamination est élevée (Fig. 2A, Fig. 2C), plus la supériorité de la souche $6 \mathrm{com}$ parée à la souche 42 s'affirme. Ce phénomène est interprété par le \% de surface malade totale sur feuilles et sur épis (chloroses et nécroses). Les périodes de dissémination sont déterminées à la fois par la maturation des pycnides et par les pluies.

\section{Variabilité observée après pression de sélection}

\section{Par rapport aux poids des grains (PMG et RPMG)}

La pression de sélection, exercée par un hôte, peut avoir pour origine son génotype, son cyto- plasme ou les deux associés. Après passage sur divers hôtes, les souches ont leur pouvoir pathogène augmenté ou diminué vis-à-vis de leurs hôtes d'origine (Tableau VIla). Ces perturbations semblent d'autant plus grandes que l'hôte de passage est éloignée du blé, comme l'indiquent les réponses obtenues après un passage sur
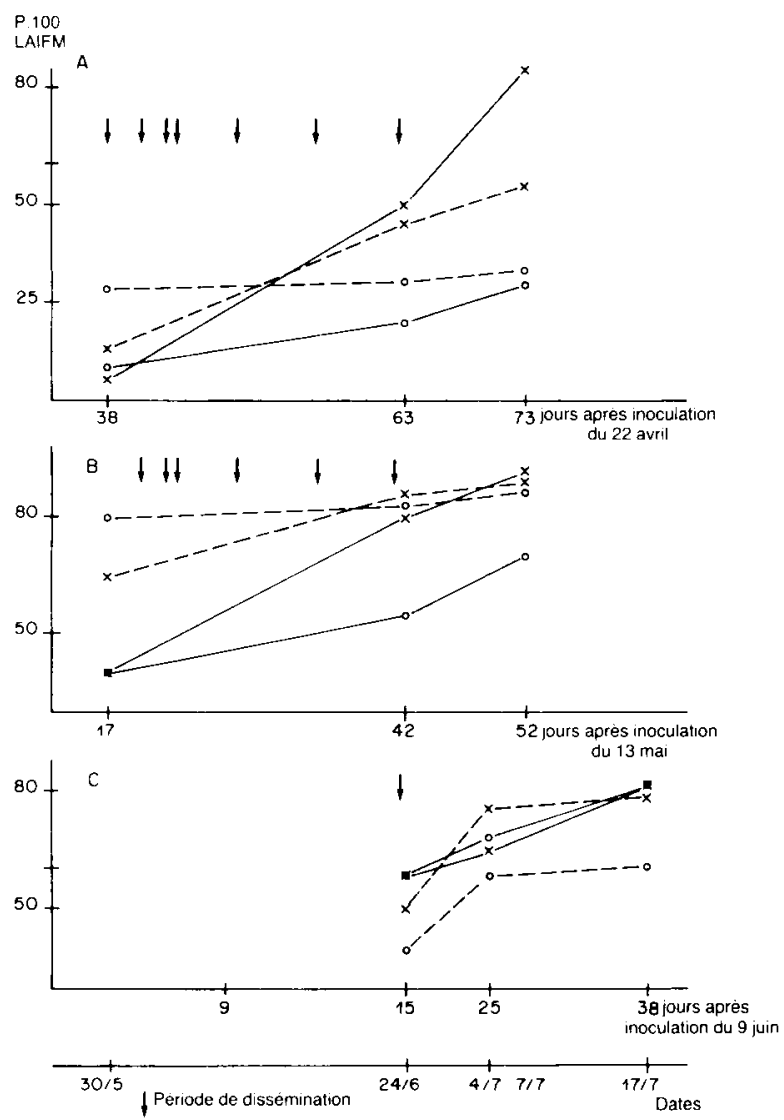

Fig. 2. Evolutions comparées d'épidémies initiées avec la souche $6(x)$ ou la souche $42(0)$ sur "Etoile de Choisy" $(--)$ et "Roazon" (- en fonction de la date de l'inoculation. 
seigle. II faut noter que le passage sur seigle , sur T. 532 ou sur la lignée de blé «B1" a entraîné, par rapport à l'absence de passage, une interaction significative hôte de passage $x$ souche $(F$ cal $=8,25)$ suivant l'hôte de lecture, ce qui introduit une inversion de classement des souches (Tableau VIIb). II est a souligner que cette inversion ne concerne que les souches d'agressivité intermédiaire.

\section{Effet du génotype de l'hôte}

A cytoplasme constant, le génotype induit des variations du pouvoir pathogène (Tableau VII). II faut relever que le seigle provoque chez la population 6 une "adaptation" à cet hôte inhabituel de Septoria nodorum.

\section{Effet du cytoplasme de l'hôte}

Cet effet du cytoplasme est perceptible en comparant les Triticale T. 832 et T. 762 (Tableau VIII). La série alloplasmique de Triticale ayant le géno- type de T. 533-8 nous a permis de comparer 3 cytoplasmes, quant aux variations qu'ils induisent en fonction du nombre de passages de la souche 6 sur des hôtes. L'effet des passages sur les 3 cytoplasmes s'exprime par la reconnaissance des chromosomes de l'hôte qui réagissent avec les 3 souches qui en résultent (Rapilly et al., 1989).

\section{Hôte de lecture différent de l'hôte de passage}

La Figure 3 illustre le RPMG de 2 variétés de blé contaminées avec des cultures ayant subi 1 à 4 passages sur cette série alloplasmique. Dès le premier passage, il y a augmentation du pouvoir pathogène. Cette augmentation se maintient, mais sans s'accroître avec les autres passages. Ce phénomène s'observe pour les 2 variétés de blé. Par ordre croissant du gain d'agressivité, les cytoplasmes peuvent se classer ainsi: T. timopheevi $>$ Secale cereale $>T$. aestivum. Toutefois, on peut relever certaines exceptions : sur «Etoile de Choisy", ce classement ne s'observe pas; il reflète la plus grande sensibilité de «Roazon».

Tableau VIla. Effet du changement d'hôte (1 passage sur limbe) sur le pouvoir pathogène de 4 souches de Septoria nodorum, apprécié par la réduction du poids de 1000 grains en \% de deux variétés de blé contaminées à l'anthèse.

Hôtes de passage

Hôtes de lecture

$$
\text { Etoile de Choisy }
$$

6

IS1

IS2

Isolats de S. nodorum
Lyon $\quad 6$

Roazon

\begin{tabular}{|c|c|c|c|c|c|c|c|c|}
\hline \multirow{2}{*}{ Pas de passage } & 17,21 & 4,68 & 1,10 & 9,98 & 14,26 & 8,1 & 4,86 & 10,49 \\
\hline & $\mathrm{a}$ & $\mathrm{c}$ & d & $\mathrm{b}$ & a & $b$ & c & $b$ \\
\hline \multirow[t]{2}{*}{ Roazon } & 22,28 & 8,45 & 3,06 & 10,30 & 15,36 & 11,21 & 4,26 & 10,56 \\
\hline & a & b & $\mathrm{c}$ & b & a & $a b$ & $\mathrm{c}$ & b \\
\hline Lignée B1 & 16,73 & 7,76 & 2,03 & 2,44 & 18,46 & 17,54 & 2,55 & 3,8 \\
\hline \multirow[t]{2}{*}{ T. $532=$ Clercal } & $\begin{array}{c}a \\
18,66\end{array}$ & $\begin{array}{c}\mathrm{b} \\
11,57\end{array}$ & $\begin{array}{l}c \\
4,15\end{array}$ & $\begin{array}{l}c \\
9,44\end{array}$ & $\begin{array}{c}a \\
16,58\end{array}$ & $\begin{array}{c}a \\
16,13\end{array}$ & $\begin{array}{l}b \\
3,21\end{array}$ & $\begin{array}{l}\mathrm{b} \\
9,55\end{array}$ \\
\hline & $\mathrm{a}$ & $a b$ & c & b & $\mathrm{a}$ & a & $\mathrm{c}$ & $\mathrm{b}$ \\
\hline \multirow[t]{2}{*}{ Seigle } & 16,49 & 9,12 & 3,16 & 4,24 & 15,44 & 16,98 & 1,22 & 4,71 \\
\hline & $\mathrm{a}$ & b & C & C & a & a & c & $b$ \\
\hline
\end{tabular}

Les lettres indiquent, pour chaque ligne, si les valeurs sont differentes entre elles au seuil de $5 \%$.

Tableau VIlb. Classement des souches à partir du Tableau VIla, par ordre décroissant du pouvoir pathogène.

\begin{tabular}{|c|c|c|}
\hline \multirow[t]{2}{*}{ Hôtes de passage } & \multicolumn{2}{|c|}{ Hôtes de lecture } \\
\hline & Etoile de Choisy & Roazon \\
\hline $\begin{array}{l}\text { Pas de passage } \\
\text { cv. Roazon } \\
\text { Lignée B1 } \\
\text { T. } 532 \text { = Clercal } \\
\text { Seigle }\end{array}$ & $\begin{array}{l}6>\text { Lyon }>\text { IS } 1>\text { IS } 2 \\
6>\text { Lyon }=\text { IS } 1>\text { IS2 } \\
6>\text { IS } 1>\text { Lyon }=\text { IS } 2 \\
6=I S 1>\text { Lyon }>\text { IS } 2 \\
6>\text { IS } 1>\text { Lyon }=\text { IS } 2\end{array}$ & $\begin{array}{l}6>\text { Lyon }=\text { IS } 1>\text { IS } 2 \\
6=\text { IS } 1>\text { Lyon }>\text { IS } 2 \\
6=\text { IS } 1>\text { Lyon }=\text { IS } 2 \\
6=\text { IS } 1>\text { Lyon }>\text { IS } 2 \\
6=\text { IS } 1>\text { Lyon }>\text { IS } 2\end{array}$ \\
\hline
\end{tabular}


Tableau VIII. Effets d'un changement d'hôte (génotype ou cytoplasme) sur la souche 6, exprimés par la réduction du poids de 1000 grains, en \%, après contamination à l'anthèse de deux variétés de blé de seigle.

\begin{tabular}{|c|c|c|c|c|c|}
\hline \multirow[b]{2}{*}{ Variété } & \multicolumn{2}{|c|}{ Hôtes de passage } & \multicolumn{3}{|c|}{ Hôtes de lecture } \\
\hline & Cytoplasme & Génotype & E. de Choisy & Roazon & Petkus \\
\hline Petkus & Secale cereale & Secale cereale & $\begin{array}{c}21,43 \\
a\end{array}$ & $\begin{array}{c}14,06 \\
b\end{array}$ & $\begin{array}{c}20,45 \\
a\end{array}$ \\
\hline T. 832 & Secale cereale & T. 533-8 & 21 & 20 & $\begin{array}{c}13,82 \\
h\end{array}$ \\
\hline T. 26 & T. timopheevi & T. 532 & $\begin{array}{l}a \\
5,28 \\
c\end{array}$ & $\begin{array}{l}\mathrm{a} \\
9,41 \\
\mathrm{c}\end{array}$ & $\begin{array}{l}b \\
-\end{array}$ \\
\hline T. 762 & T. timopheevi & T. 533-8 & $\begin{array}{c}15,19 \\
b\end{array}$ & $\begin{array}{l}9,26 \\
\mathrm{c}\end{array}$ & - \\
\hline Souche & e passage & & $\begin{array}{c}12,01 \\
b\end{array}$ & $\begin{array}{c}13,80 \\
b\end{array}$ & $\begin{array}{l}2,33 \\
c\end{array}$ \\
\hline
\end{tabular}

Les lettres indiquent si les valeurs d'une même colonne sont différentes entres elles au seuil de $5 \%$.

Hôte de lecture identique à l'hôte de passage

Sur les Figures 4 sont reportées les réductions du poids de 1000 grains enregistrées sur 4 hôtes, en fonction du nombre de passages réalisés (de 1 à 4) sur le même hôte. On constate, là aussi, dans tous les cas, un effet important du premier passage. Mais par la suite le pouvoir pathogène tend à revenir à son niveau de départ,

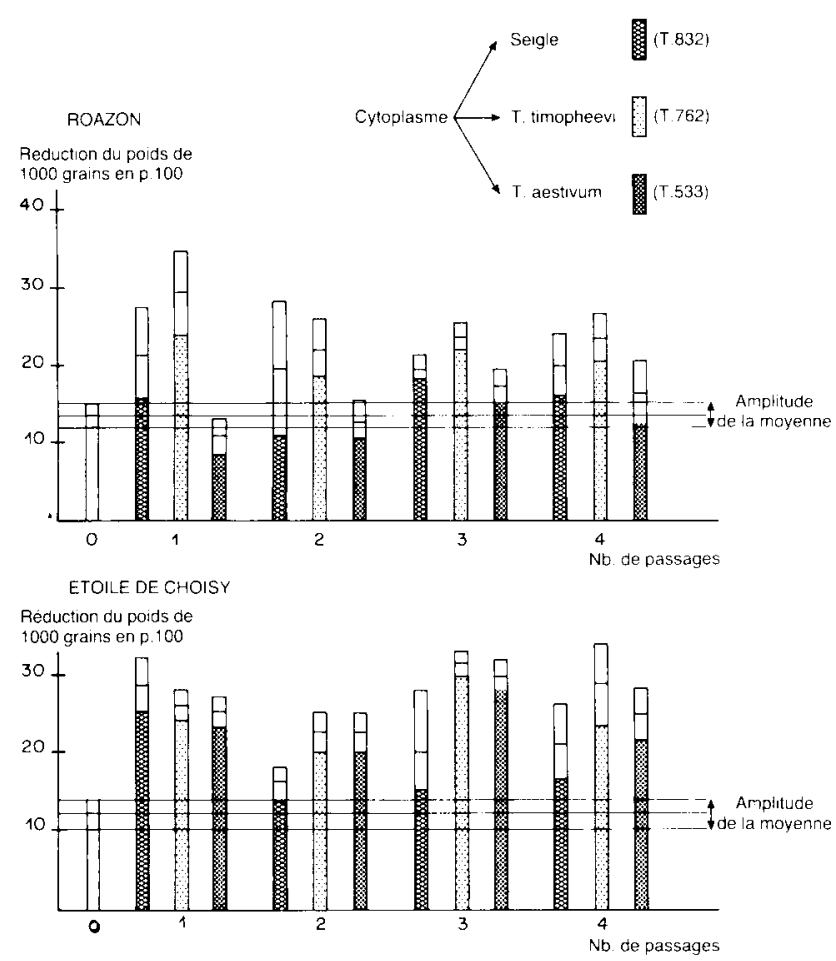

Fig. 3. Variations de l'agressivité de la souche 6, en fonction du nombre de passages sur des Triticale ayant le même génotype mais des cytoplasmes différents. Lecture par la réduction du poids de 1000 grains, après contamination à l'anthèse de deux variétés de blé: “Etoile de Choisy" et "Roazon". sauf après passages sur génotype et (ou) cytoplasme de seigle.

\section{Conséquences de ces variations sur le com- portement de lignées de blé}

Les variations subies par le parasite se traduisent par un accroissement de son agressivité sur les 2 variétés sensibles, "Roazon» et "Etoile de Choisy». Par contre, le comportement de lignées à haut niveau de résistance générale, exprimé en RPMG (Tableau IX), n'est pas modifié. La lignée B1, qui associe tolérance et certains éléments de la résistance partielle, en particulier une résistance à la contamination, donne des réponses sensiblement constantes. La lignée L22, qui associe un haut niveau de résistance partielle et une tolérance plus faible, montre des fluctuations qui sont à la limite de la signification.

\section{DISCUSSION ET CONCLUSION}

Que I'on prenne comme critère de réponse de l'hôte au parasite le poids de 1000 grains après contamination de l'épi à l'anthèse ou la vitesse d'extension des nécroses foliaires, nos résultats montrent que l'amplitude de réponse entre isolats de diverses origines est aussi grande que celle constatée entre pycnides d'une même souche ou entre monopycnidiospores issues d'une même pycnide. Le contenu d'une pycnide est donc une population faite d'individus ayant des agressivités très différentes. Tout isolat est donc une population qui a une grande plasticité.

En nous référant à des travaux précédents (Skajennikoff \& Rapilly, 1983, 1985), nous rap- 

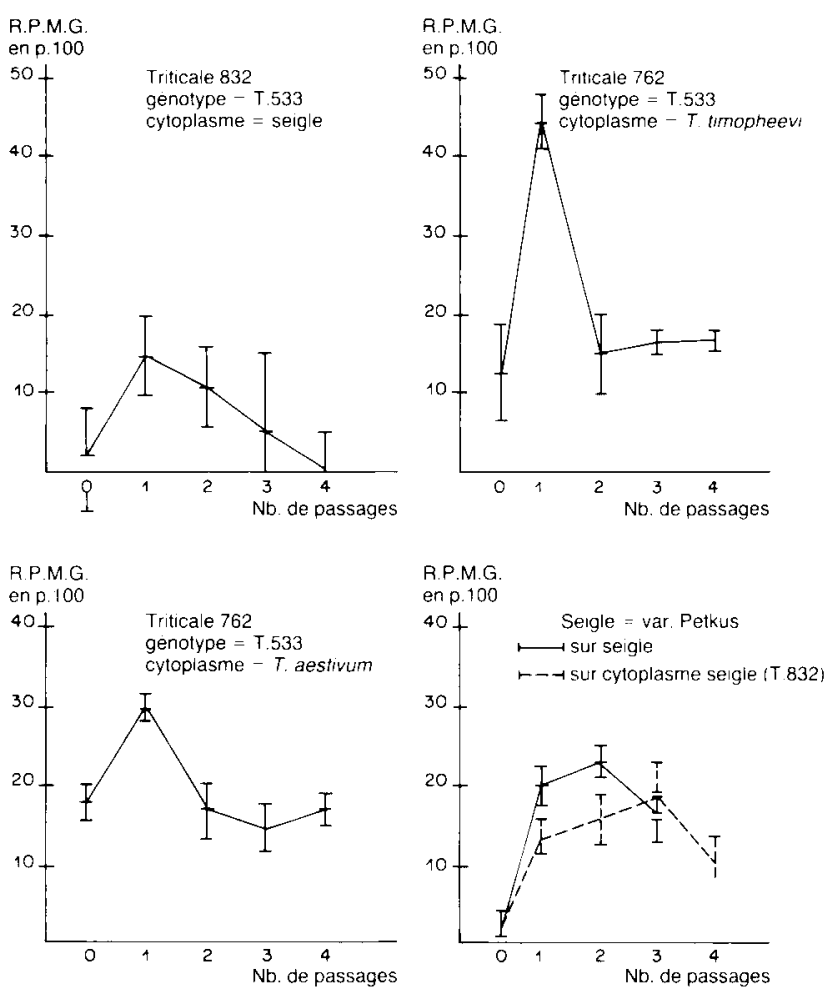

Fig. 4. Agressivité exprimée en \% de réduction du poids de 1000 grains (RPMG) de la souche 6 de $S$. nodorum, après 0 à 4 passages sur l'hôte de lecture.

Tableau IX. RPMG en \%, enregistrée après variation par changement d'hôte, de la souche 6 sur une lignée de blé tolérante, ayant un niveau moyen de résistance partielle (lignée B1), et sur une lignée moyennement tolérante, ayant un haut niveau de résistance partielle (lignée L22).

Hôtes de Hôtes de passage (de la souche 6)

lecture

Pas de

passage

$\begin{array}{llll}B 1 & L 22 & T 532 & T 26\end{array}$

\begin{tabular}{llllll}
\hline B1 & $+0,8$ & $+0,02$ & $+4,34$ & $+1,5$ & $+2,6$ \\
L22 & $-2,9$ & $+1,8$ & $-10,75$ & $-10,36$ & $-0,1$ \\
\hline
\end{tabular}

pellerons que le passage de la souche 6 sur le Triticale "Clercal» se traduit par l'apparition d'une souche qui, sur blé, réduit la durée d'incubation (LPI) et augmente la vitesse d'extension des nécroses foliaires $\left(P_{\max }\right)$. Ces deux variations permettent d'obtenir une souche aux caractéristiques très proches de l'isolat 42 , qui, lui, a été directement isolé de «Clercal».

Nos résultats sur le comportement, pour la tolérance et pour la résistance partielle, d'une série monosomique de blés contaminée avec les souches 6 ou 42 confirment que ces souches sont 2 populations associant des génotypes en partie différents (Rapilly et al., 1988b). L'associa- tion au sein d'un même cirrhe de génotypes différents est aussi confirmée par les réponses obtenues avec les cultures monospores. Dans ce cas, il n'est pas étonnant qu'un changement d'hôte «filtre" au sein de cette population les éléments qui lui sont adaptés; cette filtration pouvant s'exercer par le génotype et/ou le cytoplasme de l'hôte. II existe peu d'exemples dans la littérature où le cytoplasme permet de révéler une nouvelle race du parasite, le cas le plus connu étant le cytoplasme "Texas" du maïs et Helminthosporium maydis (Müller \& Koeppe, 1971). Plus le noyau (seigle) ou le cytoplasme (Secale cereale ou T. timopheevi) de l'hôte de passage est éloigné de celui des blés cultivés, plus la fraction de la population parasitaire réisolée après le premier passage est agressive pour ces mêmes blés et pour l'hôte de passage. Nous constatons sans pouvoir l'expliquer que, vis-à-vis du blé, le gain d'agressivité se maintient, quel que soit le nombre de passages préalables sur les différents hôtes, mais que, vis-à-vis de ceux-ci, il décroît après un maximum obtenu par un premier passage. Enfin, une variation d'agressivité, lue par la réduction du poids de 1 000 grains ou par rapport à la vitesse de progression des surfaces contagieuses, ne reflète pas la potentialité épidémique des souches. Cela est suggéré par la comparaison des épidémies initiées avec les souches 6 et 42, où il apparaît au niveau soit des disséminations, soit des contaminations ultérieures que la souche 42 est pénalisée par rapport à la souche 6 .

Les travaux d'Osbourn (1985) sur des mutants de $S$. nodorum ont bien confirmé que les noyaux d'une seule monospore sont tous identiques entre eux. Mais alors, comment expliquer que des monospores de deuxième génération, issues d'une même monospore, et ayant donc toutes la même identité nucléaire, diffèrent entre elles sur le plan de l'agressivité, comme le montrent nos résultats? L'agressivité est-elle gouvernée par le noyau seul, par le génome cytoplasmique ou par les interactions nucléo-cytoplasmique? La part du cytoplasme dans le pouvoir pathogène d'un champignon est peu étudiée, mais, dans certains cas, l'intérêt du génome cytoplasmique a été mis en évidence. Le phénomène de sénescence chez Podospora anserina est gouverné par le génome mitochondrial (Wright et al., 1982). Cependant, nous n'avons observé aucune caractéristique de sénescence apparente chez les souches de Septoria nodorum utilisées. Chez Pyricularia oryzae, Hunst et al. (1986) s'interrogent sur le rôle de l'ARN double-brin dans le pouvoir pathogène de ce parasite. Enfin, Newton (1987) a mis en évidence dans plusieurs isolats de $S$. nodorum la présence d'ARN double-brin, 
plus rarement de particules de type viral, mais il semble que ces éléments n'aient aucune liaison avec le pouvoir pathogène des souches.

La signification épidémiologique de clones nucléaires que sont les monospores est difficile à concevoir. En effet, l'unité de dissémination infectieuse de ce parasite ne correspond pas à des spores isolées, mais à de petits amas de spores véhiculés par les gouttelettes d'éclaboussures. C'est donc une population qui contamine l'hôte, et les associations nucléaires, obtenues par hétérocaryose, qui pourraient lui permettre de s'adapter à celui-ci sont rompues à chaque période de sporulation et de dissémination.

Septoria nodorum apparaît comme un parasite d'une très grande plasticité, ce qui est sans doute le reflet de sa capacité à se développer en saprophyte et en nécrotrophe. Pour mieux juger de ses facultés à se spécialiser face à des hôtes différents et à des mécanismes différents de résistance, il nous paraît indispensable d'étudier le pouvoir pathogène d'ascospores, seuls éléments du parasite issus de la fusion des noyaux.

\section{RÉFÉRENCES}

Bronnimann A. (1968) Zur Kenntniss von Septoria nodorum Berk. dem Erreger der Spelzenbraüne eine Blättdure des Weizens. Phytopathol Z. 61, 101-146

Cauderon Y., Cauderon A., Gay G. \& Roussel J. (1985) Alloplasmic lines and nucleo-cytoplasmic interactions in triticale. In: Genetics and Breeding of Triticale, EUCARPIA meeting, Clermont-Ferrand (France) 2-5 July 1984. - INRA Paris, 177-191

Cunfer B.M. \& Youmans J. (1983) Septoria nodorum on barley relationships among isolates from several hosts. Phytopathology 73, 911-914

Halama P. \& Lacoste L. (1987) Obtention contrôlée des périthèces de Leptosphaeria nodorum Müller. Soc. Franc. Pathol. Rennes, Nov. 87 (Poster)

Hunst P.L., Latterell F.M. \& Rossi A.E. (1986) Variation in double stranded RNA from isolates of Pyricularia oryzae. Phytopathology 76, 674-678

Krupinsky J.M. (1982) Comparative pathogenicity of Septoria nodorum isolated from Triticum aestivum and Agropyrom sp. Phytopathology 72, 660-681

Müller R.J. \& Koeppe D.E. (1971) Southern corn leaf blight: susceptible and resistant mitochrondria. Science 173, 67-69

Newton A.C. (1987) Occurence of dsRNA and viruslike particles in Septoria nodorum. Trans. Brit. Mycol. Soc. $88,113-116$

Newton A.C. \& Caten C.E. (1985) Heterokaryosis and heterokaryon incompatibility in Septoria. In: Septoria of Cereals (A.L. Sharen Ed.) 13-15. U.S.A. Dept. of Agricul, A.R.S. 12

Osbourn A.E. (1985) Host adaptation and variation in Septoria nodorum. Ph D. Univ. of Birmingham, Dept. 07 Genetics, U.K.

Osbourn A.E., Scott P.R. \& Caten C.E. (1986) The effects of host passaging on the adaptation of Septoria nodorum to wheat or barley. Plant Pathol. 35, 135 145

Rapilly F. \& Delhotal P. (1986) Sur la durabilité de résistances partielles à Septoria nodorum Berk. chez le blé ( $T$. aestivum L.). Etudes prospectives réalisées par la simulation. Agronomie 6, (4), 325-336

Rapilly F., Foucault B. \& Lacazedieux J. (1973) Etudes sur l'inoculum de Septoria nodorum Berk. (Leptosphaeria nodorum Müller) agent de la septoriose du blé: l. les ascospores. Ann. Phytopathol. 5 (2), 131-141

Rapilly F., Auriau P., Laborie Y. \& Depatureaux C. (1984) Recherches sur la résistance partielle du blé tendre à Septoria nodorum. Agronomie 4, (7), 639-651

Rapilly F., Auriau P., Cauderon Y., Richard H. \& Depatureaux C. (1988a) Réactions à la contamination par Leptosphaeria nodorum Müll. des feuilles, épis et grains de plantes $F_{2}$ issues du croisement entre une lignée de blé résistante et la série monosomique de Courtôt. Agronomie 8, (7), 647-652

Rapilly F., Auriau P., Richard H. \& Depatureaux C. (1988b) Réaction d'une série monosomique de blé à la contamination des feuilles et des épis par deux souches de Septoria nodorum Berk. (Leptosphaeria nodorum Müller) Agronomie 8 (9), 801-809

Rapilly F., Richard H., Skajennikoff M., Cauderon Y. \& Rousselle J. (1989) Pressions de sélection exercées par le noyau ou le cytoplasme de l'hôte sur l'agressivité d'un pathogène nécrotrophe du blé: Septoria nodorum Berk. Agronomie 9 (7), 703-718

Scott P.R. (1973) Incidence and effect of Septoria nodorum on wheat cultivars. Ann. Appl. Biol. 75, 321 . 329

Shaw D.E. (1953) Cytology of Septoria and Selenophoma spores.. Proc. Linnean Soc. N.S.W. 78, 122130

Skajennikoff M. \& Rapilly F. (1983) Etudes sur l'agressivité de Septoria nodorum Berk. Effets des hôtes (triticale et blé) et des organes attaqués. Agronomie 3, (2), $131-140$

Skajennikoff M. \& Rapilly F. (1985) Consequences of Triticale introduction on the parasitic fungi on wheat. In : Genetics and Breeding of Triticale, Eucarpia meeting, Clermont-Ferrand (France) 2-5 July 1984. - INRA Paris, 537-549

Trottet M., Dosba F. \& Doussinault G. (1975) Conséquences d'une attaque de Septoria nodorum Berk. sur la formation du grain chez $T$. aestivum L. et chez Aegilops ventricosa Tausch. Incidences en sélection. Ann. Amélior. Plant. 3, 131-140

Wright R.M., Horrum M.A. \& Cummings D.J. (1982) Are mitochondrial structural genes selectivity amplified during senescence in Podospora anserina? Cell. 29, 505-515 